Bull. Mater. Sci., Vol. 22, No. 3, May 1999, pp. 647-655. (C) Indian Academy of Sciences.

\title{
On the relevance and requirements of biomaterials
}

\author{
R SIVAKUMAR \\ Biomedical Technology Wing, Sree Chitra Tirunal Institute for Medical Sciences and Technology, \\ Poojapura, Trivandrum 695012 , India
}

\begin{abstract}
The technological orientation of current medical practice is reflected in the effective utilization of a number of diagnostic and therapeutic devices. Synthetic and natural biomaterials alone or in combination form the basis for development of such devices that are in contact with different tissues. The effect of materials on tissues and vice versa needs to be understood to ensure safety and effectiveness of the devices. This calls for biological evaluation of materials and devices, in addition to a spectrum of recommended toxicological testing of materials depending upon the duration and the type of tissue in contact with. Besides being non-toxic, the material is required to meet the functional requirements with the appropriate host response which is termed as biocompatibility.

This paper reviews the evolution and the requirements of biomaterials. The in vitro and in vivo evolution methodologies are highlighted based on our experience in developing blood bag, dental composite, hydroxyapatite and fibrin glue. The requirements of biomaterials in the current context of advances in the fields of tissue engineering and biomimetics is outlined.
\end{abstract}

\section{Introduction}

The current medical practice is driven by technological advancements for identification, cure and prevention of diseases. The devices that are used for the above range from disposable ones like blood bag, IV set, wound dressing to a variety of permanent implants that include intraocular lens, heart valve, total hip joint etc. About 2500 medical devices and an equal number of diagnostic products are in the market (Szycher 1996). Approximately 40 different materials are in current use covering a range of applications. The material can be of synthetic or natural origin, which interfaces temporarily or permanently with viable tissues. Table 1 lists some of the biomaterials that are used for different medical devices. When the interaction between the biomaterial and the living tissue evokes acceptable biological response, the material is considered biocompatible. The response of the living system can be localized to areas near the material/tissue interface or systemic in nature. In the time domain, the reaction can be acute (short term) or a chronic one.

The selection and development of a candidate material is guided by the functional requirement of the device. For example, the total hip joint replacing the bones has to transmit and distribute the body load and stress in addition to articulation. Stainless steel, cobalt alloys, titanium, alumina, ultra high molecular weight polyethylene are some of the materials used for different components like femoral stem, head and acetabular cup. Polymethylmethacrylate (PMMA), hydrogel and foldable soft acrylics are the materials of choice for intraocular lens (IOL) which needs to transmit light.
Thus, the biocompatibility requirements of materials intended for medical device applications encompass both the non-toxicity and the functionality and are invariably application-specific.

\subsection{Evolution of biomaterials}

Materials in medicine date back to ancient civilizationsIndia, China, Egypt etc-where the medicine was practiced as part of the religious and mystical activities. Metallurgical tools were used for surgical treatment, tendons for suture etc. The first implanted material was gold plate for cleft palate in 1588. Till the polymer industry was developed in 1950s, the metallic materials like silver, platinum, stainless steel and cobalt base alloys were mainly used. The first synthetic polymer to be used as a biomaterial was polymethylmethacrylate (PMMA) for dentures. Further details on the history of biomaterials can be found in the compiled lecture notes on "Biocompatibility-History, practice and perspectives".

During the $40 \mathrm{~s}$ and 50s, knowledge regarding the material-tissue interaction was at its infancy. This, coupled with the fact that there was a low demand, resulted in surgeons trying out the industrial and other available materials as substitutes for impaired tissues in the body. Faced with different kinds of biological reactions such as inflammation, irritation etc, the polymers were synthesized with a minimum amount of additives and with the highest possible purity to achieve non-toxicity. Table 2 lists some of the additives that can elicit unfavourable biological reactions leading to complications. 
With improved knowledge, a number of toxicological evaluation tests were developed for plastic drug containers and later extended to other materials.

In addition to the toxicological tests, the long-term performance was derived partly from suitable animal experiments, followed by controlled clinical trials in humans. The above approach has led in some cases to different grades of materials for biological applications. For example, in the case of silicone, three grades were evolved viz.:

- Clean grade: All quality control (QC) procedures and good manufacturing practice (GMP) followed

- Medical grade: All biological testing have been carried out in addition to QC plus GMP

- Implant grade: In addition to above, two year implant data is available

Table 1. Types of biomaterials.

\begin{tabular}{|c|c|}
\hline Polymers: & $\begin{array}{l}\text { Acrylics, silicons, polyurethanes, teflon, polyester, } \\
\text { polylactic acid, polyglycolic acid }\end{array}$ \\
\hline Metals: & Stainless steel, cobalt alloys, gold, titanium alloys \\
\hline Ceramics: & Alumina, glasses, hydroxyapatite, carbons \\
\hline Composites: & Ceramic coated materials, ceramic in polymer matrix \\
\hline Biological: & $\begin{array}{l}\text { Porcine heart valves, bovine bone, auto and allo } \\
\text { grafts }\end{array}$ \\
\hline
\end{tabular}

Table 2. Toxic compounds related to polymers.

\begin{tabular}{|c|c|}
\hline Compounds & Remarks \\
\hline Monomers & Not polymerized, depolymerized \\
\hline Initiators & Intact and decomposed \\
\hline Catalyst & Cross linking, curing etc \\
\hline Additives & $\begin{array}{l}\text { Antioxidents, plasticizers, UV absorbants, } \\
\text { lubricants, pigments, antistatic agents }\end{array}$ \\
\hline Others & Byproducts, degradation products \\
\hline
\end{tabular}

In spite of these precautions and long history of the use of silicone as a biomaterial in a number of applications (maxillofacial and skeletal implants, hydrocephalus shunt etc), the recent reports on rupture of breast implants have led to the possible link between silicone gel and autoimmune disease. This reminds us of the limitations of our knowledge regarding the complex and dynamic nature of biological environment. There is hence a need for caution in extending the successful use of a material from one application to another. The form of material (size, bulk or powder, dense or porous), the surface morphology, the type of tissue in contact with and the duration have considerable influence on the nature of material-tissue interaction. Table 3 gives a description of the material shapes along with a few of the process technologies utilized to form the shapes.

Biomaterials are constantly interacting with natural dynamic living tissues. Hence, the interface plays a critical role in determining the functionality. A number of surface modified biomaterials are used in a variety of applications such as:

- Catheters

- Vascular grafts

- Orthopedic and dental implants

- Implantable electrodes

- Separation membranes

- Contact/intraocular lenses and

- Cell cultureware

The surface is generally altered to overcome the deterioration in performance or failure of biomaterials due to degeneration, fracture, infection, systemic effects etc. The areas of application for surface modification can broadly be divided into:

- Tissue fixation (e.g. tissue valves)

- Antimicrobial surface (urinary catheters)

- Low friction surface (pacing leads, catheters)

- Thrombo resistant surface (vascular grafts)

Table 3. Shapes of biomaterials.

\begin{tabular}{lll}
\hline Form & \multicolumn{1}{c}{ Process } & \multicolumn{1}{c}{ Product } \\
\hline Sheet tube & Molding, extrusion & Blood bag, catheters \\
Porous tube & Weaving, knitting, stretching & Vascular grafts \\
Hollow fibres & Extrusion with phase inversion & Oxygenator, dialyser \\
Threads & Fibre spinning & Sutures \\
Porous shapes & Sintering, conversion of coral & Bone grafts \\
Spring & Wire drawing, coiling & Stents, guide wire \\
\hline
\end{tabular}


There are a number of techniques such as chemical modification of polymers using UV light and plasma, ion beam techniques to alter the structure (texturing) or chemistry (implantation of $\mathrm{N}$ ions) or both. These technologies aim to alter the surface properties like hydrophobicity, hydrophilicity, protein binding, cell/ molecular affinity and thereby influence biocompatibility (Wise et al 1995).

\section{Qualification of biomaterials}

There are no implantable materials per se, but only materials with acceptable biocompatibility to meet the functional requirements of different applications. The ASTM standards (1998) have published specifications and guidelines related to medical materials and devices, covering physical, chemical and mechanical properties and also biological evaluation. There are also many ISO standards which have been adopted by Bureau of Indian Standards (BIS) (1994) covering the above.

The question that often arises is, "How to qualify a biomaterial?" or, "Is there a qualified biomaterial available in the market for designing medical devices?" The mechanistic interpretation of biocompatibility calls for acceptable performance of the material without any adverse effect on cellular and tissue functions that it is in contact with and also on the rest of the physiological functions such as immune systems. Then such a material qualifies as a biomaterial.

The biocompatibility testing is carried out

- To identify any adverse reactions that can lead to failure of the material or to unacceptable clinical disease

- To determine if devices made out of new materials or processes functions as intended under simulated use conditions and

- To advance new technologies.

Table 4. Raw material characterization-Phase 1 .

Chemical

- Extractables/leachables

- Physicochemical tests as per pharmacopoeia

- IR, chromatography

- Trace elements

Physical

- Mechanical properties (Before and after environmental tests)

- Surface characterization

Biological

- Cytotoxicity and hemolysis
The standard industrial practice regarding the safety evaluation of medical devices consist of four phases. These are (i) screen/quality raw materials, (ii) material biocompatibility (ISO and other guidelines), (iii) product/ process validation and (iv) release/audit testing.

Table 4 lists some of the physico-chemical characterization of materials and the first level biological screening tests that are carried out. The classification of devices for evaluation of material biocompatibility along with typical examples under each category is given in table 5 .

The biological evaluation for determining the biocompatibility consists of studying the possible adverse reactions arising out of direct contact of or the leachables/ extractables from the materials in the physiological environment.

The areas of concern are cytotoxicity, sensitization, irritation, systemic toxicity, tissue response to implantation, genotoxicity, haemocompatibility etc. The rationale behind these tests and the critical issues in assessing biocompatibility of devices are well covered in the literature (Silver and Doillon 1989; Gad 1997).

In table 6 , a few of the tests designed to characterize the finished product and to validate the manufacturing process are listed. These qualification studies are intended to demonstrate that manufacturing processes control are sufficient for preproduction quality assurance and product specifications. Testing is carried out to verify the effectiveness of such controls and to evaluate the biological effects of processing aids added during manufacture.

The phase 4 (table 7) consists of release and audit testing of finished products on a lot and periodic basis to assure product uniformity and compliance with label claims and good manufacturing practice (GMP) requirements.

Table 5. Device classification for material biocompatibilityPhase 2.

Surface devices

- Skin (sutures, electrodes)

- Mucosal membrane (urinary catheter)

- Breached surfaces (burn dressing)

Externally communicating

- Blood path, indirect (blood administration)

- Tissue/bone communicating (dental filling materials)

- Circulating blood (oxygenators)

Implant devices

- Tissue/bone (HIP implant)

- Blood (vascular grafts) 
The current status of understanding is such that, it is not possible to apply a rigid set of test protocols and pass/fail criteria. The test results are to be applied with interpretation and judgement by appropriate professionals qualified by training and experience taking into account of the factors relevant to the device/materials, intended use and the current knowledge base about the material and device, based on the scientific literature and previous clinical experience (Braybrook 1997).

Realizing the complexity of testing and meaningfully interpreting the data, ISO has prepared a draft document on Medical Devices-Risk Management, which includes risk analysis based on identification of the hazards and the consequences thereof. Standard techniques like failure mode and effect analysis (FMEA) and fault tree analysis (FTA) can be utilized for probabilistic safety analysis.

\section{Biomaterial/device development-Our experience}

Our institute has been involved in the development and commercialization of medical devices and implants. Facilities and expertise have been developed for characterization and evaluation of biomaterials and devices. During the developmental phase, the materials and devices need to undergo specialized testing with respect to the intended application. These are illustrated by a few examples based on the experience gained during the development of blood bags, dental composites, bioceramics and fibrin glue.

Table 6. Product/process validation-Phase 3 .

Sterility

- Validation of sterilization cycle

- Bioburden

Safety

- Pyrogen, LAL

Chemical

- Chemical residues

- Particle analysis

\section{Environmental control}

- Viable and nonviable particulate analysis

- Microorganism identification

- Environmental monitoring

Process control

- Process water system validation

- Device cleaning process qualification

\subsection{Blood bags}

These are single use disposable units used for collection, component separation and transfusion of blood. It is made of non-toxic formulation of plasticized polyvinyl chloride for flexibility.

In addition to the material toxicological tests, a number of functional tests have been specified in ISO standard 3826 (1993). These are (i) air content, leakage test, (ii) thermal stability $\left(-80\right.$ to $\left.+50^{\circ} \mathrm{C}\right)$, (iii) vapour transmission, (iv) vinyl chloride monomer $(<1 \mu \mathrm{g} / \mathrm{g})$ and (v) extractable plasticizer content.

The objectives of the tests are to minimize risks resulting from microbiological contamination, air embolism, blood-container interaction etc. It has been found that the requirements of optimum storage conditions for red cells and plasma differ from that of platelets. Platelets can be stored upto 3 weeks at about $25^{\circ} \mathrm{C}$ under gently agitating conditions. The above requirement can be achieved by specifically developing platelet bags with higher oxygen permeability with appropriate plasticizer.

There are a few concerns regarding the use of PVC in blood bag. One of them is the leaching of phthalate plasticizer into the blood (Blass 1990). It has been shown in animal model that the phthalate has adverse biological reactions. Based on the existing data, it is generally accepted that this is a problem during repeated use of PVC tubes as in dialysis. There are blood bags formulated with alternate plasticizers like citrates as well as from polyolefines. Waste disposal of PVC, especially, the toxic emissions like dioxines on incernation is yet another concern, that has led to investigations on alternate materials, preferably biodegradable. An economically viable and acceptable solution is yet to emerge.

\subsection{Dental composites}

Dental composites belong to the class of aesthetic restorative materials to fill cavities, build up broken tooth, bond orthodontic brackets etc. These must have adequate strength and wear resistance to withstand the

Table 7. Routine testing-Phase 4.

Release/periodic audit testing

- Endotoxin content (LAL test)

- Pyrogenicity

- Sterility

- Cytotoxicity

- Material characterization

- Particulate testing

- Bioburden testing 
masticating and the biting forces. One of the main life limiting factors is the marginal leakage of food and bacteria through the gap between the composite and enamel/dentine due to inadequate bonding (figure 1).

Dental composites consist of two major components viz. a resin matrix based on bisphenol A-glycidyl methacrylate (bis-GMA) and quartz or glass inorganic filler with additives for activating the polymerization. Colouring pigments are also added to aesthetically match the shade with the natural tooth. The fillers impart strength, wear resistance and also reduce the shrinkage of the composite during in situ polymerization in the oral environment. A number of bonding agents have been developed to ensure integration of the restorative with the tooth (Miyazaki et al 1996).

Table 8 outlines a brief description of the American dental Association's (ADA) acceptance programme for the composite adhesives, which include laboratory, biological and clinical evaluations.

\subsection{Bioceramics}

The use of bioceramics for artificial bones has arisen mainly due to better matching of mechanical properties with bones, as compared to metals. Dense polycrystalline alumina was introduced in 1970s as dental and hip implants. Its low fracture toughness and strength prompted the use of partially stabilized $\mathrm{ZrO}_{2}$. Stringent processing

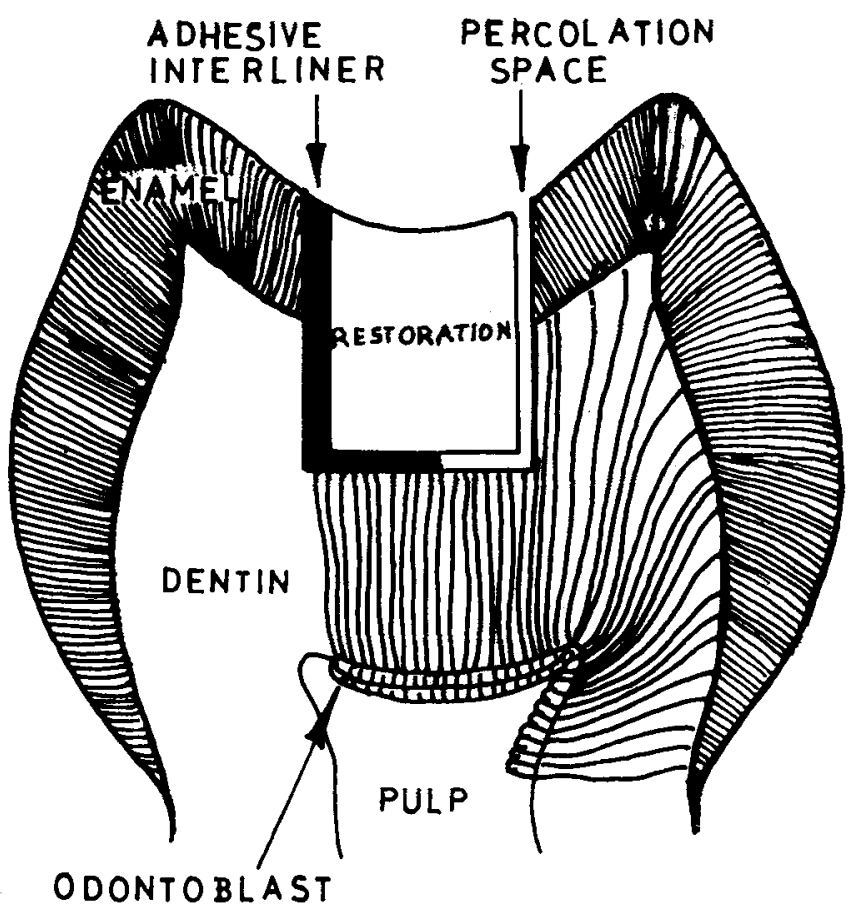

Figure 1. Schematic cross-section of tooth with a restorative filled cavity. requirements coupled with difficulties in ensuring complete reliability have limited their applications.

Among the bioceramics, the calcium phosphate materials are the best choice, as their chemical composition is close to the mineral phase of the bone. This results in complete integration with bone. Calcium phosphate based ceramics have been under development and use since 1976. Unlike the bioinert ceramics of $\mathrm{Al}_{2} \mathrm{O}_{3}$ and $\mathrm{ZrO}_{2}$, these are bioactive and so new bone is formed on the surface of these ceramics. They are used in the form of powders, porous blocks, dense shape and as porous coatings on metallic implants. The porous nature has attracted much attention, as it has been shown that the new bone growth velocity can be increased when the open pore size is above $100 \mu \mathrm{m}$. There are different types of phosphates depending on the $\mathrm{Ca}$ to $\mathrm{P}$

Table 8. ADA acceptance programme (Dentin and enamel adhesives).

1. Laboratory evaluation

Setting time (if applicable)

Colour stability and colour generating reactions

Dentin bond strength (shear or tension)

- Tooth storage condition

- Hydration state of Dentin

- Surface preparation and presence/absence of smear layer

Enamel based strength

Microleakage testing

2. Biological evaluation

ANSI/ADA document 41

\section{Clinical evaluation}

Efficiency of caries free class IV erosion/abrasion

At least 30 restorations $/ 2$ independent studies $/ 6$ months for provisional \& 18 months for full acceptance

Restorations per patient

Patient age

Clinical site characterization

Records

- Patient's particulars

- Primers or pretreatment including acid etching

- Methods of insertion (bulk, incremental)

- Finishing techniques/materials

Evaluation parameters

- Document the procedures

- Training of clinical evaluator for compliance with procedures

- Methods of data recording (e.g. intraoral colour slides)

- Data on retention, marginal discolouration, stressful occlusion, post-operative sensitivity etc.

- Impressions at base line and recall appointments 
ratio, but the stoichiometric hydroxyapatite (HA) and beta tri-calcium phosphate (TCP) have been investigated extensively and applications in dental and orthopedic fields have been developed.

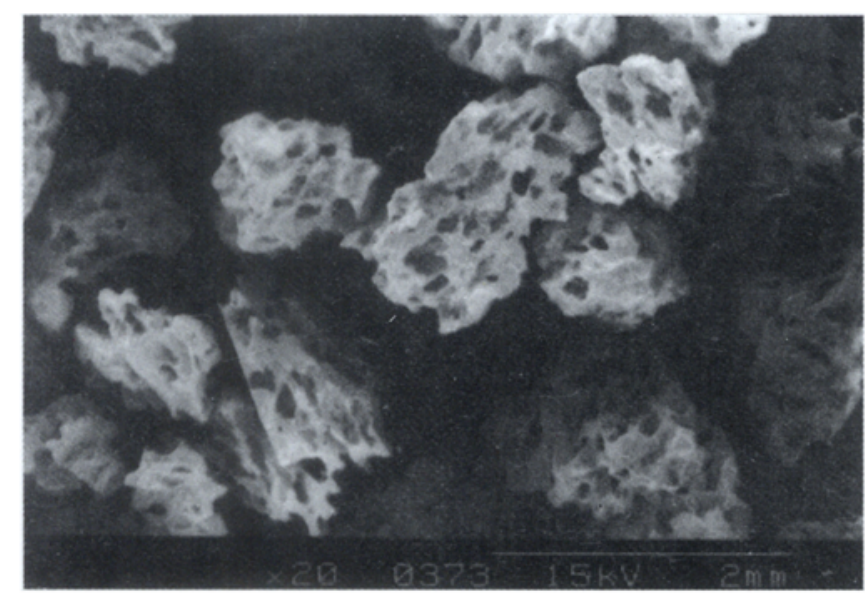

Figure 2. SEM of morphology of porous hydroxyapatite granule.
Our institute's programme includes the development of hydroxyapatite (HA), bioglass (BG) and HA-BG composites for bone graft applications (Sivakumar 1998). Figure 2 shows a typical photomicrograph of porous granules synthesized in our laboratory. The material needs to be characterized in terms of chemical analysis, trace elements, density, pore size (in case of porous granules/blocks) and strength followed by toxicological evaluation.

For specific applications, it is necessary to evaluate the efficacy in terms of functional evaluation. In the case of periodontal injury, in addition to the growth of new bone, it is necessary to regenerate the periodontal ligament. It is not possible to clinically assess this functional requirement as histopathological evaluation on cut-sections is required. Hence, the granules were tested using rabbit and dog as animal models.

The histology of implantation of HAP granules in periodontal defect in rabbits for six months can be seen in figure $3 \mathrm{a}$. The defect is completely repaired by well-formed bone. Periodontal collagen fibres are observed between the newly formed bone and the repaired cementum. There is a mild epithelial downgrowth. This

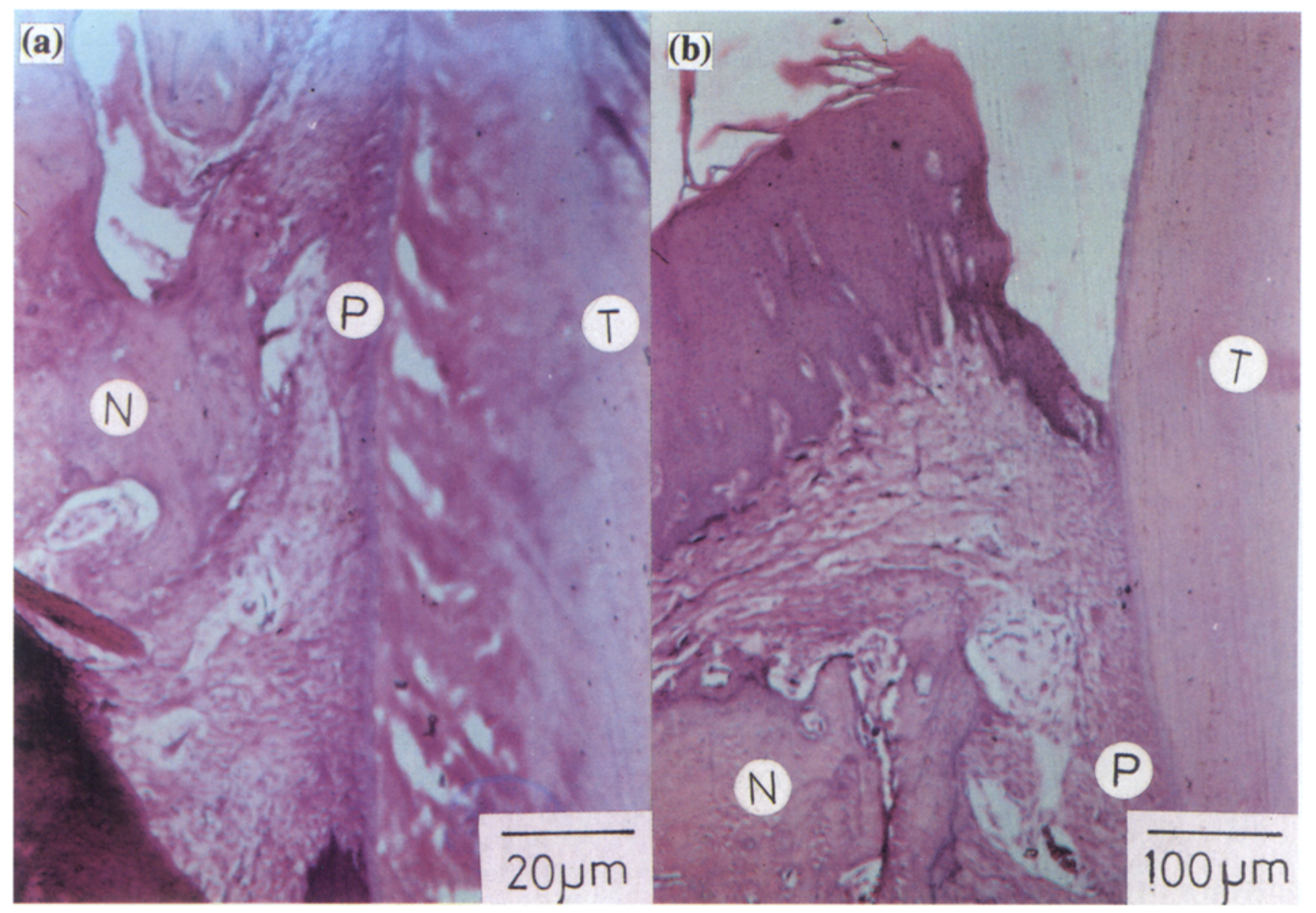

Figure 3. Histopathology of bone formation in the periodontal defect in rabbit (6 months). (a) Filled with HA granule and (b) unfilled defect-control ( $\mathrm{N}$, new bone; $\mathrm{P}$, periodontal ligament; $\mathrm{T}$, tooth). 
is better compared to the healing seen in the control defect without granules (figure $3 b$ ), where the connective tissue can be observed along with the newly formed bone. The periodontal ligament, though formed is not well oriented as in the case of the defect filled with HAP granule.

\subsection{Fibrin glue}

Tissue adhesives are attractive options to replace sutures and wires in closing wounds and repairing soft and hard tissues. Fibrin glue or sealant is a biological adhesive derived from blood, simulating the final stages of coagulation cascade. Fibrinogen from blood plasma is converted to insoluble fibrin network in presence of thrombin and calcium ion (Silver et al 1995; Sierra and Saltz 1996; see also the special issue on Fibrin $1998 \mathrm{~J}$. Long Term Effects Medical Implants 8). Its adhesive properties, hemostatic activity and positive effect on angiogenesis, wound healing and biodegradability have resulted in a wide range of applications in almost all major surgeries.

Being a biological product, the plasma is to be derived from blood already screened for hepatitis B, HIV and for a variety of metabolic disorders. For large scale preparation, plasma from different blood donors may have to be pooled, in which case viral deactivation procedures have to be standardized and validated by spiking with known quantities of different types of viruses.

In addition to optimizing the concentration of components and ensuring purity, the tensile and shear adhesive strengths are to be assessed. As part of the ongoing development, the tensile adhesive strength was determined by applying load on 1 square $\mathrm{cm}$ skin graft which was glued back onto anaesthetized rat (figure 4). The failure occurred around $65 \mathrm{~g} / \mathrm{cm}^{2}$ comparable to similar glue available in the market. The functional evaluation of the glue was carried out by performing different types of surgeries as documented in table 9.

\section{Future scenario}

The development of biomaterials has so far progressed based on the feedback received from the biological evaluation of materials and devices in simulated laboratory tests, experimental animal models and clinical evaluation. Further growth along these lines will continue for a reasonable number of years. Concurrently, increased biological understanding at cellular and subcellular levels is giving rise to revolutionary approaches that are expected to change the biomaterial scenario. An outline of these developments is given below.

The improved knowledge base generated in material-tissue interaction is leading towards design of materials, wherein, the required biocompatibility can be controlled in a predictable way for different applications. Availability of well characterized materials and surfaces with a data base on in vitro and in vivo performance of different devices would help in validating the design and materials. Innovations in process technologies like rapid prototyping would help in reducing the developmental period and the cost.

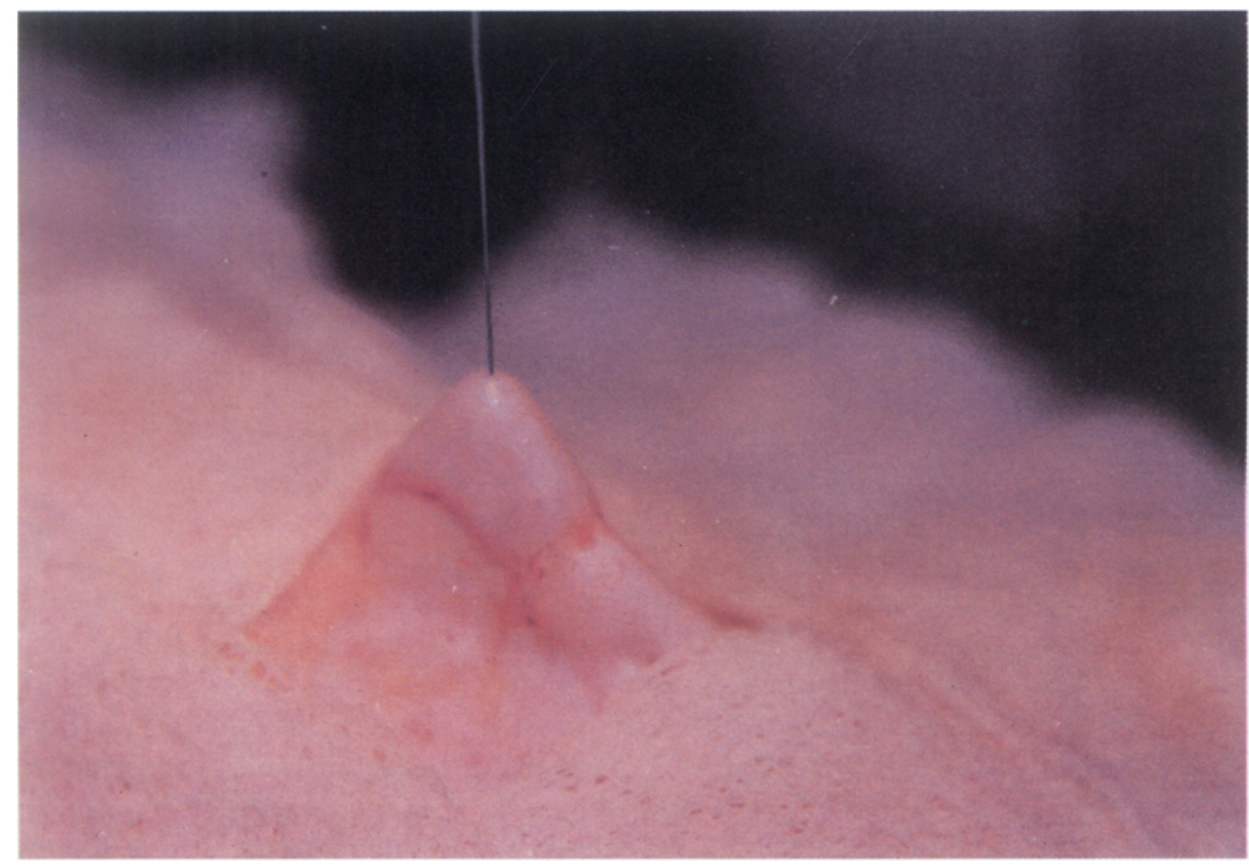

Figure 4. Measurement of tensile strength of fibrin glue. 
Table 9. Functional evaluation of Fibrin glue.

\begin{tabular}{|c|c|c|}
\hline Application & Animal and technique & Results \\
\hline Vascular surgery & $\begin{array}{l}\text { Dog; Vascular grafts and patch } \\
\text { anastamoses }\end{array}$ & $\begin{array}{l}\text { Good sealing of anastomosis, blood loss from the suture holes } \\
\text { stopped completely }\end{array}$ \\
\hline Neurosurgery & Rabbit; Dural closure & Dural closure was complete. No CSF leak \\
\hline Ophthalmic surgery & $\begin{array}{l}\text { Rabbit; Stab injured cornea was } \\
\text { sealed with fibrin glue }\end{array}$ & $\begin{array}{l}\text { The aqueous humor formed in } 24 \mathrm{~h} \text {, and normal eye ball } \\
\text { appearance restored }\end{array}$ \\
\hline Lung & $\begin{array}{l}\text { Dog; Stab injured lung was sealed } \\
\text { and inflated with ventilator }\end{array}$ & $\begin{array}{l}\text { Haemostasis and complete sealing of air leak, } 2 \text { min after glue } \\
\text { application }\end{array}$ \\
\hline Skin graft & $\begin{array}{l}\text { Rat; } 1 \mathrm{~cm}^{2} \text { skin graft was appo- } \\
\text { sitioned without suture }\end{array}$ & $\begin{array}{l}\text { Good adhesion between the injured tissue surfaces, no skin } \\
\text { retraction }\end{array}$ \\
\hline Liver & $\begin{array}{l}\text { Rat; Stab injured liver, sealed with } \\
\text { glue }\end{array}$ & $\begin{array}{l}\text { Bleeding was arrested immediately, bonding of injured tissue } \\
\text { surface was good }\end{array}$ \\
\hline
\end{tabular}

The changes in medical practice such as minimally invasive surgeries are catalyzing the development of new miniature devices that are collapsible and amenable to guidance through endoscopic procedures.

Besides these incremental improvements, recent advances in biomimetics and tissue engineering would have a direct impact on the development of future biomaterials. Biomimetic implies a close reproduction of a biological material (Calvert 1996). Structural biological materials range from soft gels to hard ceramics. Our understanding of the formation of complex architectures in nature is slowly improving. In nature, the various macromolecules are involved in nucleation, growth and manipulation of inorganic phases during the synthesis of mineralized biological materials. The most important aspect of biomineralization is the achievement of an intimate association of inorganic and organic phases, starting from nanoscale to macrolevels. Even though 'mimicking' the complex biological environment in the laboratory is rather difficult, it is possible to divide the process into stages that can be adopted to practical processing schemes. A composite component can have regions of pure ceramic and pure polymer with gradients of properties in between without interfaces. Such a part can be built up, layer by layer, similar to multilayer ceramics or by stereolithography techniques. These structures need to have aspects of intelligence and self-repair. The emerging approaches of nano- and micro-fabrication as applicable to biology have been covered (Hoch et al 1996). Such a biomimetic approach is also being pursued to develop materials for engineering and other applications.

Tissue engineering is a new interdisciplinary science that focuses on the development of biological substitutes at the scale of tissue or organ. It is achieved by using isolated cells or cell substitutes, growth factors and cells seeded on or within matrices. A wide range of tissues like bone, cartilage, enamel, dentin, oral soft tissues, cornea, skin and liver are being developed. Cellular engineering is also being pursued to develop new diagnostic and therapeutic devices by exploring the inherent capabilities of biological cells. Many of the products rely on a biomaterial component. The material can act as a scaffold or matrix, as an immunoisolation barrier or as a means to deliver active protein, gene or cell product through controlled degradation. The biomaterial can also have an active long-term interaction as a load bearing or bulking component.

The emerging fields of cellular and tissue engineering have raised concerns regarding their safety and efficacy. The products consist of cellular components in conjunction with natural or synthetic biomaterial. Omstead (1998) has provided a summary of the factors that need to be considered during the design and developmental stages. These cover different points as applicable to the unique nature of the tissue-engineered products. They are cell and tissue sourcing, biomaterial characterization, testing, quality control assurance, non-clinical and clinical evaluation. For instance, it is not possible to terminally sterilize products containing living cells or tissues. It is hence necessary to ensure the safety of cells prior to their incorporation. Specific in vitro assays are required to assess cell identity, purity and potency. The cell identity would involve specifications on morphology, expression of antigens and genes.

The future approaches that have direct bearing on the development of biomaterials can be summarized as (i) scientific basis for determining performance and quality of implants, (ii) biologically based material design and (iii) advanced processing and manufacturing.

Current research on materials designed for cell, drug and gene based therapies can lead to control of cell functions. Such an ability to control specific biological activities has the potential to replace many of the medical devices in current use. The developmental challenges of 
such materials would be considerably different from the current issues pertaining to integration of synthetic materials to natural tissue. The ultimate success lies in developing methodologies for tissues to repair themselves based upon the nature's ways of responding and solving problems in the complex, yet fascinating world of living.

\section{References}

Annual Book of ASTM Standards 1998 Medical devices and services (PA: Am. Soc. for Testing Materials)

Biocompatibility-History, practice and perspectives 1991 in Biocompatible surfaces (Lancaster, PA: Technomic Pub. Co.)

Blass C R 1990 in Progress in biomedical polymers (eds) C G Gebelein et al (New York: Plenum Press)

Braybrook J H (ed.) 1997 Biocompatibility assessment of medical devices and materials (Sussex: John Wiley \& Sons)

Bureau of Indian Standards 1994 Biological evaluation of medical devices-Part I, Guidance of selection of tests IS12572
Calvert P 1996 in Processing of ceramics, Materials science and technology series (ed.) R J Brook (Weinheim: VCH Publishers) Vol. 17B, p. 51

Gad S C (ed.) 1997 Safety evaluation of medical devices (New York: Marcell Dekker Inc.)

Hoch H C et al (eds) 1996 Nanofabrication and biosystems (Cambridge: Cambridge University Press)

Miyazaki M et al 1996 Biomed. Mater. \& Eng. 615

Omstead D R 1998 Tissue Eng. 4239

Sierra D and Saltz R 1996 Surgical adhesives and sealantsCurrent technology and applications (Lancaster, PA: Technomic Pub. Co.)

Silver F and Doillon C (eds) 1989 Biocompatibility (New York: VCH Publishers) Vol. 1

Silver F H et al 1995 Biomaterials 16891

Sivakumar R 1998 Indian J. Orthopedics 33131

Szycher M 1996 J. Biomater. Appl. 1176

Wise D L et al (eds) 1995 Encyclopedic handbook of biomaterials and bioengineering, Part A: Materials (New York: Marcel Dekker Inc.) p. 785 